\title{
Opinion on Prospective Application of Self-Healing Materials in Orthopedic Prostheses
}

\author{
Hamed Hosseinzadeh ${ }^{1 *}$ and Hamid Seyyedhosseinzadeh ${ }^{2}$ \\ ${ }^{1}$ Henry M Rowan College of Engineering, USA \\ ${ }^{2}$ Orthopedic Research Group, USA \\ *Corresponding author: Hamed Hosseinzadeh, Henry M Rowan College of Engineering, USA
}

\begin{tabular}{|c|c|}
\hline ARTICLE INFO & ABSTRACT \\
\hline Received: 豐 April 09, 2019 & Citation: Hamed Hosseinzadeh, Hamid Seyyedhosseinzadeh. Opinion on Prospective \\
\hline Published: 慧 April 22, 2019 & $\begin{array}{l}\text { Application of Self-Healing Materials in Orthopedic Prostheses. Biomed J Sci \& Tech Res } \\
\text { 17(2)-2019. BJSTR. MS.ID.002970. }\end{array}$ \\
\hline
\end{tabular}

\section{Opinion}

Hip and knee joint replacements are among the most successful surgeries in the medicine and their application is growing very fast. This fast-growing procedure still has some limitations, the most important of which is its limited life in the human body. This limitation may be due to several factors. Some of them like periprosthetic fractures or traumatic failures are not directly related to the prostheses. But some of them like infection, wear, corrosion and microcracks leading to gross failure are closely related to the prosthetic materials and related design. The orthopaedic literature has reached to an acceptable maturity in prosthetic designing. But material science has a long way to go in this field. Recently, material scientists have paid much attention to produce some self-repairing/self-healing materials to prolong the lifetime of some materials in long-lasting products such as bridges, space crafts, airplanes and body implants. Self-healing materials could play a very important role for orthopaedic implant technology which is heavily based on the development and use of biomaterials. In general, orthopaedic implants are non-living materials (e.g. metals, polymers and ceramics) and comprise several different biomaterials working together in modular parts, such as in a total hip replacement system which may contain up to 4 or more different materials such as titanium, cobalt-chrome alloy, polyethylene and polymethylmethacrylate (PMMA or bone cement) [1]. The failure mechanisms of orthopedic prostheses are not so fast growing, so we do not need very fast repairing mechanisms.

Self-healing materials can be divided into two different classes, depending on the required trigger and the nature of the self-healing process: Non-autonomic and autonomic. Non-autonomic selfhealing materials require a modest external trigger, like heat or light.
In contrast, autonomic self-healing materials do not require any additional external trigger; the damage itself is the stimulus for the healing [2]. Despite great advances in joint implant technology, the ultimate grail of joint implant surgery has yet to be achieved: a truly long-lasting implant in a young active individual. Current problems that contribute to implant failure include the major conundrums of failure of the bone-implant interface (lack of osseointegration) and implant infection [1]. Since the current prosthetic materials are not a living tissue, they may fail after a certain time of operation and once they begin to fail, this process propagates very fast. If a microcrack happens in a high stress zone of the prosthesis, since the stress continues, it concentrates at that specific point and propagates the microcrack into a complete fissure, the same as what happens in fracture of the neck of the femoral stems. Or when a corrosion occurs, there will be an aggravated process of corrosion which eventually leads to gross failure of the prosthesis, the same as what occurs in the trunnions of the large femoral head prostheses.

But wearing maybe the most common and important mechanism of prosthetic failure. During wearing, some part of a material in the prosthesis is removed, so the contact surface between the two surfaces is changed, leading to stress concentration and more wearing, eventually ending in prosthetic failure. This is the main mechanism of polyethylene failure. Corrosion, wearing, osseointegration factors that could show the quality of implant are happening at surface of implant in contact to human body. Many novel techniques such as using porous materials, anti-bacterial coating, metal/polymer/ceramic on metal wearing contact and etc were introduced to implant fabrication and technology to prevent failure and enhancing quality of designed implant. Although these techniques were partially successful but more refinement 
in designing is necessary to decrease negative problems of using implants and increasing for safe usage of implant for longtime. Some of these mentioned techniques would solve a problem but would make a new problem i.e. implant with metal on metal wear surface has lesser wear in comparison to polymer on metal surface but it would cause more metal ion release [1].

So, it is very important to use new designing techniques. Selfhealing quality at the surface of implants would be very helpful to prevent the formation and happening of micro cracks (fatigue/ corrosion cracks), corrosion [3] and induced corrosion due to micro cracks, wear debris and etc. Up to now, there has been no successfully commercialized application of the self-healing materials in orthopedic surgery to prolong the lifetime of the prosthesis. Offered fabrication technique of self-healing prosthesis is using microcapsules and encapsulation for polymeric and ceramic part of medical prosthesis i.e. self-heal bone cement. Femur and

\section{ISSN: 2574-1241}

DOI: 10.26717/BJSTR.2019.17.002970

Hamed Hosseinzadeh. Biomed J Sci \& Tech Res

This work is licensed under Creative Commons Attribution 4.0 License

Submission Link: https://biomedres.us/submit-manuscript.php knee prostheses are mainly comprised of metallic materials then polymeric and ceramic materials. Although designing a relevant selfhealing orthopaedic prosthesis means having self-healing metallic materials, self-healing metallic biomaterials for orthopaedic prosthesis is still very challenging. For ambient temperature, electrochemical processes would be wise and dominant strategy to design self-healing metallic biomaterials.

\section{References}

1. Wang W, Ouyang Y, Poh CK (2011) Orthopaedic Implant Technology: Biomaterials from Past to Future. Ann Acad Med Singapore 40(5): 237 244.

2. Brochu ABW, Stephen L Craig SL, William M Reichert (2011) Self-healing biomaterials. J Biomed Mater Res A 96(2): 492-506.

3. Hamdy AS, Möhwald IDH (2011) Intelligent self-healing corrosion resistant vanadia coating for AA2024. Thin Solid Films 520(5): 16681678.

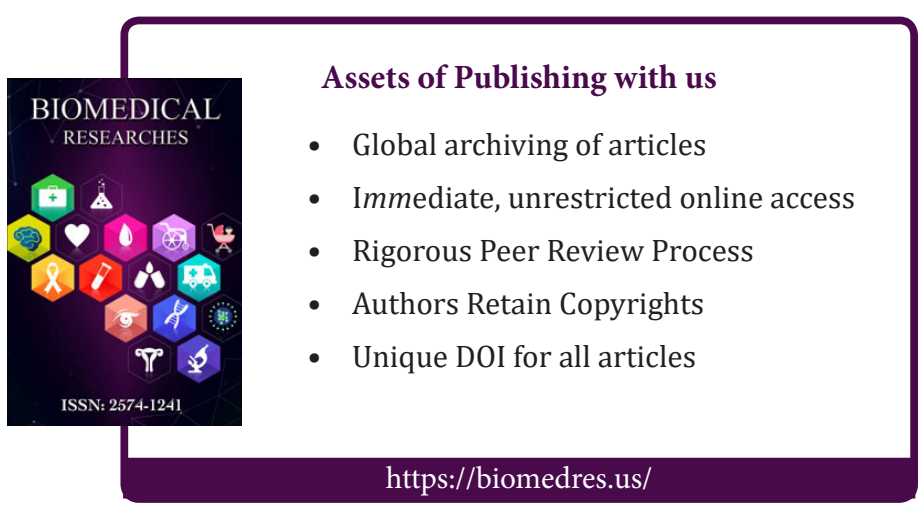

